\title{
JAXA's Optical Observation Facility for Space Debris Observation and Its Activities
}

\author{
By Toshifumi YanagisawA ${ }^{1)}$, Hirohisa KUROSAKI ${ }^{1)}$ and Atsushi NAKAJIMA ${ }^{1)}$ \\ ${ }^{1)}$ Japan Aerospace Exploration Agency, Chofu, Japan
}

(Received July 15th, 2009)

\begin{abstract}
The innovative technology research center of JAXA is developing observation technology for GEO objects in order to deal with the space debris problem. The center constructed a space debris observation facility at Mt. Nyukasa, Nagano in 2006. The observation facility contains two telescopes and two large CCD cameras. The main objective of the facility is to establish technologies to detect un-cataloged GEO debris and determine their orbits, and to incorporate theses technologies in the Bisei Spaceguard Center in Okayama. GEO debris detection software is being developed that can detect unresolved objects in CCD frames, and a new orbital determination method is being tested that can determine the orbits of many GEO objects. This paper presents the details of the facility and research activities.
\end{abstract}

Key Words: Space Debris, Optical Observation

\section{Introduction}

Since Sputnik, the world's first successful satellite, was launched in 1957, many satellites have been launched and the number of artificial objects in orbit has been increasing. Explosions and collisions of these objects create large volumes of space debris. For the safety of people and satellites, this debris needs to be detected and cataloged. Some ten million pieces of space debris larger than $1 \mathrm{~mm}$ are believed to exist but, only about 12 thousand such pieces have been cataloged.

In geostationary (GEO) orbit, the situation is serious because the minimum size of catalogued objects is about 1 meter. Breakups near this orbit were reported in 1978, 1992, and 1994, ${ }^{1)}$ and now this debris is spread over a wide longitude range in GEO, increasing the danger of secondly collisions. An observation system is required for GEO debris smaller than one meter in diameter to reduce the risk of such events.

The Japan Aerospace Exploration Agency (JAXA) has been studying observation technologies as part of its research

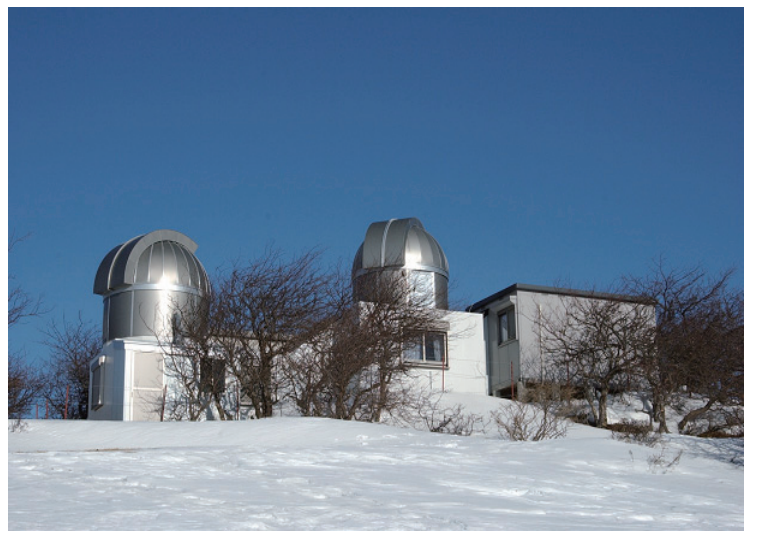

Fig. 1. JAXA's Mt. Nyukasa optical observation facility.

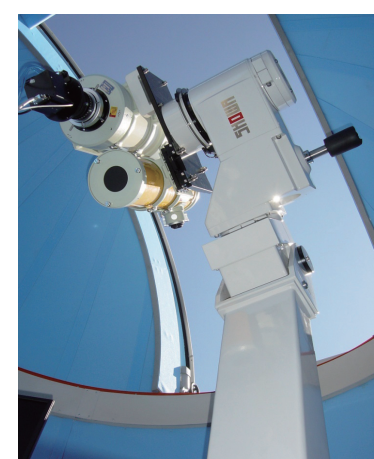

Fig.2(a). Eccentric elbow-type mount, $25 \mathrm{~cm}$ and $16 \mathrm{~cm}$ telescopes.

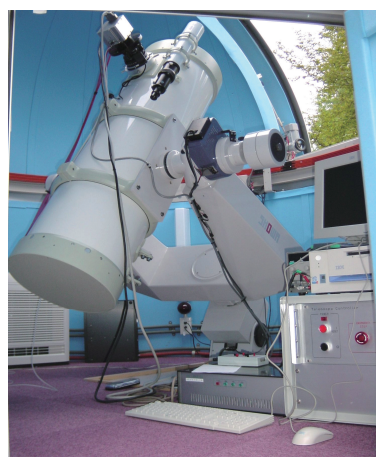

Fig.2(b). Fork-type mount and $35 \mathrm{~cm}$ telescope activity on GEO debris problems. There has been a special focus on developing image processing techniques to detect small GEO debris ${ }^{2-4)}$. The National Institute of Information and Communication Technology has also been studying observation technologies, especially those related to orbit determination of GEO debris ${ }^{5-8}$. The objective of our research is to establish practical technologies for detection and orbit determination of un-cataloged GEO debris. For this purpose, JAXA's Innovative Technology Research Center constructed an optical observation facility at Mt. Nyukasa, Nagano in November 2006. After new telescopes, CCD cameras, and analysis software were installed, the routine operation for the development of space debris observation technique has started. The details of the facility, the CCD cameras and the observation software are described in sections 2, 3 and 4, respectively. The analysis methods and orbit determination techniques that are being developed and tested are discussed in sections 5 and 6. Other research activities are shown in section 7 .

\section{JAXA's Mt. Nyukasa Optical Observation Facility}




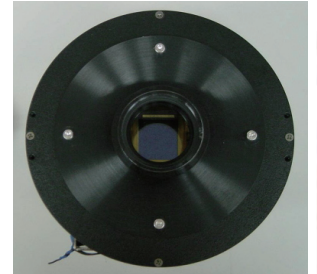

(a) Top view

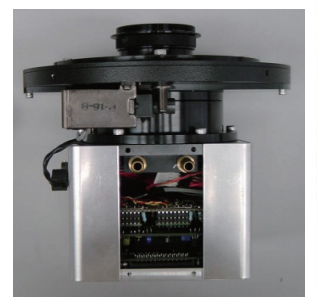

(b) Side view

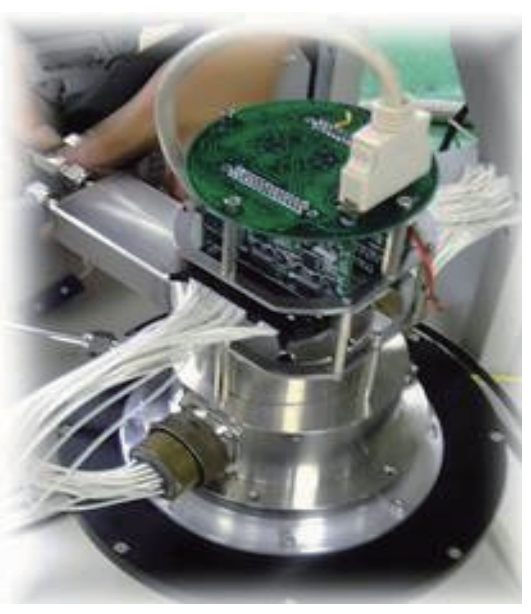

Fig.4. $4 \mathrm{~K}$ by $4 \mathrm{~K}$-camera.

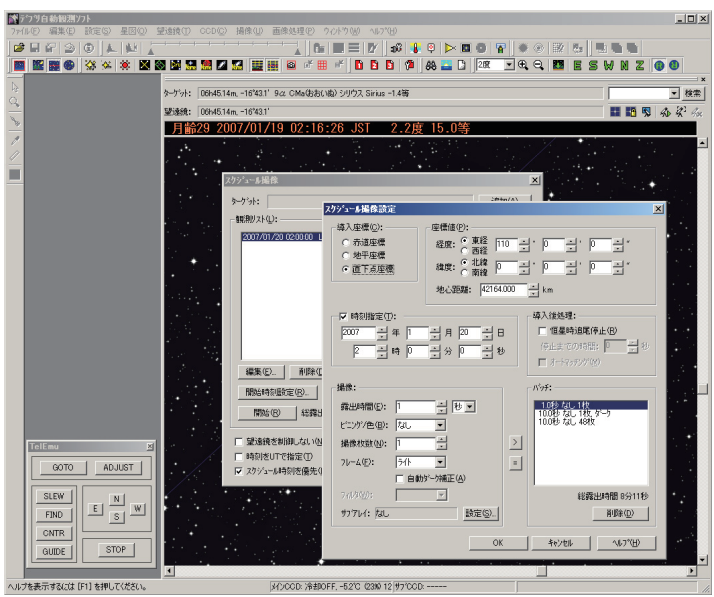

Fig.5. Debris auto-observation software.

Fig. $3.2 \mathrm{~K}$ by $2 \mathrm{~K}$-camera.

The Mt. Nyukasa optical observation facility, with a relatively small $35 \mathrm{~cm}$ telescope as its main equipment, was built for the development of observation technologies for high-altitude space debris such as that in geostationary (GEO) or geo-transfer (GTO) orbits. The main objective of the facility is to develop detection technique for space debris less than $10 \mathrm{~cm}$ in size. Current our goal is the detection of GEO space debris as small as $20 \mathrm{~cm}$ with the $35 \mathrm{~cm}$ telescope. Although a $1 \mathrm{~m}$ telescope is usually required to detect $10-20$ $\mathrm{cm}$ (20th magnitude) objects in GEO, sophisticated image processing enables us to detect objects of this size with a much smaller $35 \mathrm{~cm}$ telescope. Applying such processing to the data taken with a $1 \mathrm{~m}$ telescope makes possible the detection of GEO debris less than $10 \mathrm{~cm}$ in size.

The site is located at an altitude of $1870 \mathrm{~m}$ above the sea level, which provides an outstanding optical environment. The site can detect dark asteroids of $22^{\text {nd }}$ magnitude with the 35 $\mathrm{cm}$ telescope. Fig. 1 shows an overview of the facility, which consists of two domes, a control room, and two habitats. One dome is for a $35 \mathrm{~cm}$ telescope, the other, a $25 \mathrm{~cm}$ telescope. Fig. 2 shows the observational equipment in the domes. An eccentric elbow-type equatorial mount that can hold two 25 $\mathrm{cm}$ telescopes is shown in Fig.2 (a). A fork-type equatorial mount supporting a $35 \mathrm{~cm}$ telescope is shown in Fig.2 (b). This telescope has contributed to the discovery of many new asteroids.

\section{CCD Cameras}

Three different format CCD cameras are used in this facility, a $1 \mathrm{~K}$ by $1 \mathrm{~K}$-, a $2 \mathrm{~K}$ by $2 \mathrm{~K}$-(Fig. 3 ) and a $4 \mathrm{~K}$ by $4 \mathrm{~K}$-camera (Fig.4). All the cameras contain back-illuminated CCD chips that have high quantum efficiencies at optical wavelengths. The $1 \mathrm{~K}$ by $1 \mathrm{~K}$ - and the $2 \mathrm{~K}$ by $2 \mathrm{~K}$-cameras are cooled by Peltier devices down to 30 degrees below room temperature. The $4 \mathrm{~K}$ by $4 \mathrm{~K}$-camera is cooled down to $-100{ }^{\circ} \mathrm{C}$ by circulating refrigerant. Readout times for these cameras are fairly fast ranging from 4 to 10 seconds. As the typical exposure time is from a few seconds to 10 seconds for GEO debris observation, a fast readout time is required. The fields of view of the $2 \mathrm{~K}$ by $2 \mathrm{~K}$-camera with the $35 \mathrm{~cm}$ telescope, and of the $4 \mathrm{~K}$ by $4 \mathrm{~K}$-camera with the $25 \mathrm{~cm}$ telescope are $1.3 \times 1.3$ degrees and $2.4 \times 2.4$ degrees, respectively.

\section{Observation Software}

Each telescope and its CCD camera are controlled by automatic debris-observing software. This software manages scheduled observations that specify coordinates of field of view, start times for exposures, exposure times, exposure mode, and number of CCD frames to be taken. The software executes observation automatically throughout one night once it is set up. Fig.5 shows a screenshot of the observation software. To determine the orbit of detected GEO debris with adequate precision, a measurement accuracy of $10 \mathrm{msec}$ is required for the times of observation. The CCD camera system has a function to record exact timing of the exposure with GPS. The open and close times of the shutter are recorded in the image header with the unit of 0.001 second.

\section{Analysis Methods}

This section describes two analysis methods that are used at the Mt. Nyukasa optical observation facility.

\subsection{The stacking method}

The stacking method uses multiple CCD images to detect very faint objects that are undetectable on a single CCD image. We have been developing this image processing with validation from observations of GEO debris since $2000^{2,3)}$. The general idea of this method is described below, and details are available in the references.

As shown in Fig.6, sub-images are cropped from many CCD images to follow the presumed movement of space debris. A median image of all the sub-images is then created. In this method, photons from the space debris arrive on the 


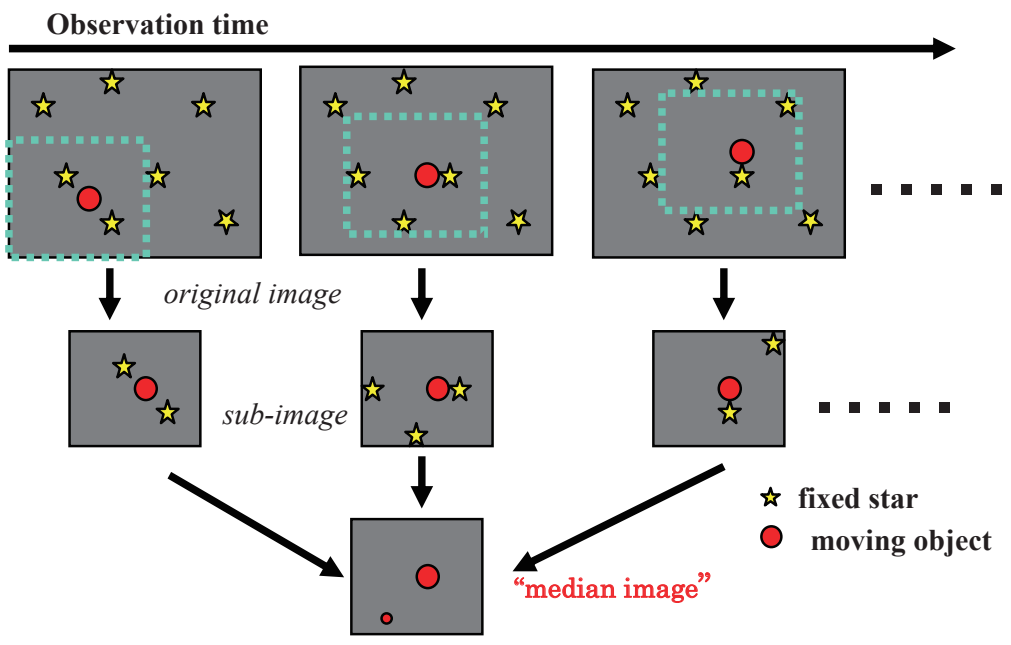

Fig.6. Stacking method.

same pixels of the sub-images, and field stars are removed by taking the median, because they appear in different places on each sub-image. Fig.7 shows an example of an asteroid detected using this method. Fig.7 (a) shows a part of one CCD image, and Fig.7 (b) shows the same region of the final image after the process was carried out using forty images. It is impossible to confirm the presence of the asteroid in Fig.7 (a), whereas the asteroid is bright and no field stars are shown in Fig.7 (b). So far, about 350 asteroids have been newly discovered by using this method with the $35 \mathrm{~cm}$ telescope. The average brightness of the discovered asteroids is about $20.5^{\text {th }}$ magnitude. This is about 2 magnitudes fainter than normally detectable by this size telescope. This discovery of many new asteroids has proven the effectiveness of this method, which enhances the detection ability of the $35 \mathrm{~cm}$ telescope to equal that of a $1 \mathrm{~m}$ telescope.

The only weak point of the stacking method is the time required to analyze the data when detecting an unseen object whose movement is not known. As mentioned before, the method presumes the movement of the object. Only when the object movement matches to the presumed one, the unseen object is detected as shown in Fig.7. Therefore, many movements must be presumed to find the right one, which is very time consuming. Although main-belt asteroids and cataloged space debris whose movements can be estimated in some way are easy targets to detect, finding near-Earth objects and un-cataloged space debris is not really practical. Using many PCs in parallel to reduce analysis time may be one solution. However, a hardware system purpose-built for this method, such as a field programmable gate array (FPGA) would be the best solution for use with an upcoming large-format CCD camera. As an FPGA is expected to reduce analysis time dramatically, efforts to translate this method to an FPGA system are underway at JAXA.

\subsection{Line-identifying technique}

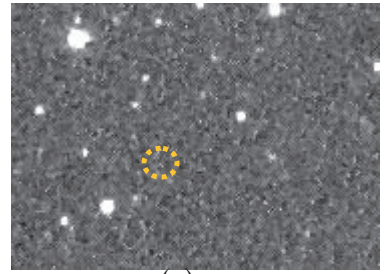

(a)

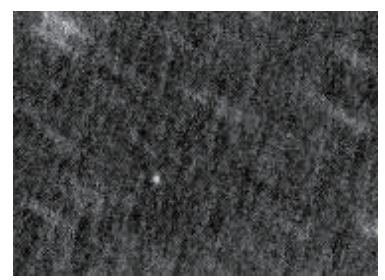

(b)

Fig.7. Asteroid detected by the stacking method.
We developed the line-identifying technique to complement the stacking method. Fig. 8 sketches the technique, and the details will be described in the near future. The line-identifying technique uses many CCD frames, as the stacking method does. First, it detects candidate objects (black dots in Fig.8) using a threshold and a shape parameter. Then, it finds any series of objects that are arrayed on a straight line from the first frame to the last frame. Appearing on a straight line as shown in Fig. 8 means that an object is moving across the field of view at a constant velocity. Using this technique enables us to detect near-Earth asteroids and unknown space debris, whose movements are unpredictable. The technique does not need to presume any particular target trajectory, as the stacking method does. The number of calculations depends on the number of candidates. For example, a commercial PC (DELL Precision 450) is able to analyze 17 frames with 400 candidates in each frame in 7 minutes, which is quite

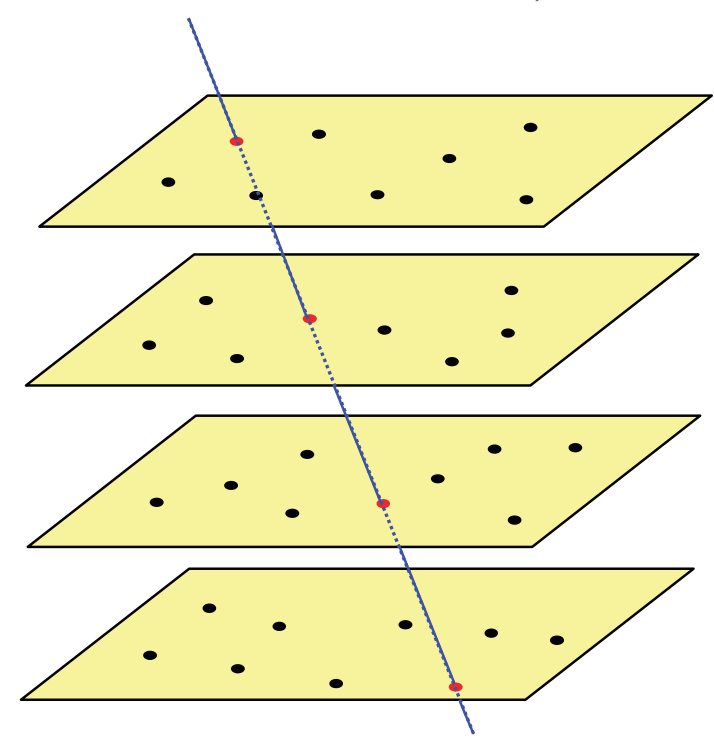

Fig.8. Line-identification technique 


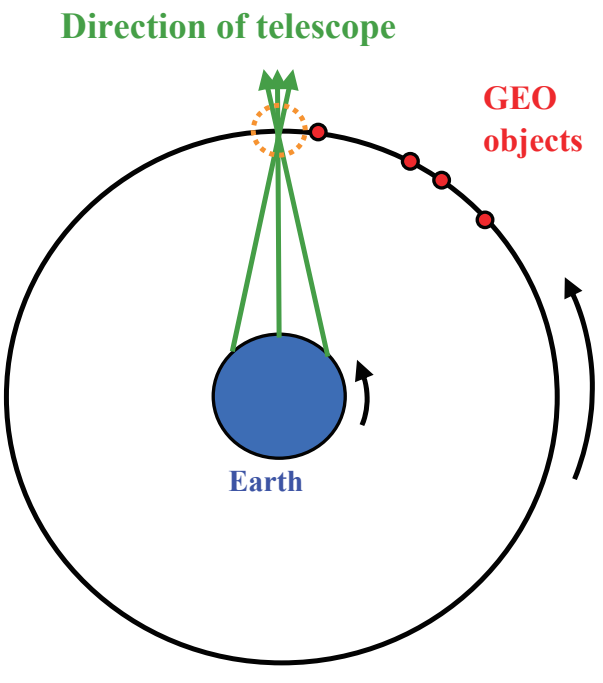

Fig. 9. Observation of one inertia position.

acceptable. The user can select an appropriate number of candidates in each frame by considering the capability of the PC and the number of frames. With sufficient computing power, the number of candidates can be increased by lowering the detection threshold, meaning that darker objects will be detectable.

Although the line-identifying technique works efficiently in a practical analysis time, its ability to detect faint objects does not measure up to the stacking method. Its analysis time increases exponentially with the number of candidates in each frame. The same problem arises with the stacking method, but since line-identifying can cope with this by reducing the number of candidates, that technique will be used to analyze the actual observational data while the FPGA system is being developed for the stacking method.

\section{Orbit Determination Technique}

After detection of space debris, the orbit of the detected target must be determined. A reliable orbit determination requires a long observation arc. However, the narrow field-of-view (FOV) of optical telescopes makes it difficult to re-acquire the same object after a few hours, especially in the case of eccentric orbits. To get a long arc, a telescope must follow one target for a long period of time. Therefore, determining the precise orbits of many GEO-crossing objects is very time-consuming, and is not an efficient use of telescope time. Umehara invented an observation method that sights numerous bits of space debris efficiently, using one telescope to cope with this situation ${ }^{7,8)}$. The method utilizes a fundamental principle of orbital mechanics - an object in a bound orbit always returns to the same orbit location after one complete revolution, if perturbation is negligible. This means if a telescope observes one specific inertia position for two nights, an object that passed through the field-of-view of the telescope in the first night must do so again in the second night (Fig. 9). From two nights' data, this method determines an approximate orbit that is accurate

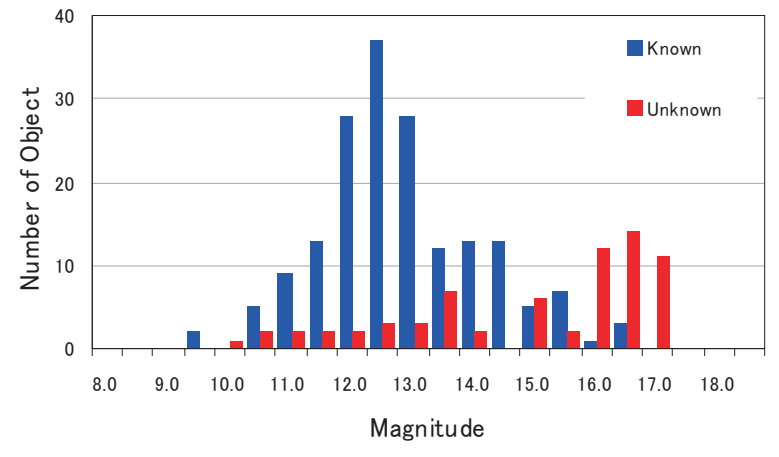

Fig. 10. Brightness distribution of cataloged (blue column) and un-cataloged (red column) objects.
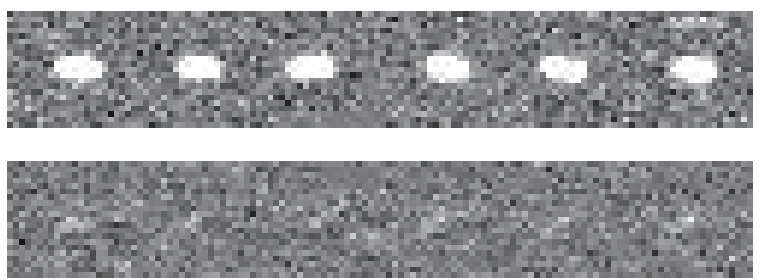

Fig. 11. Images of cataloged (upper) and un-cataloged (lower) objects that appear in data from the campaign. One is $12^{\text {th }}$ magnitude and the other is $17^{\text {th }}$ magnitude.

enough to predict the position of the target on the third night. Precise orbits are determined from three nights' data. Readers will find more detail on this method in the references.

We carried out a test observation to evaluate this method at the Mt. Nyukasa optical observation facility. One inertia position where a great deal of space debris was expected to pass through was monitored for three hours on each of two nights. On the first night, 20 objects were detected, and 18 on the second. Twelve objects were identified as twice-observed by comparing the elements of their presumed-circular orbits. For these objects, the positions on the third night were estimated from approximate orbits calculated from two nights' data. All 12 of the objects were detected on the third night. Precise orbits were determined, and the standard deviations of o-c values (observed position - calculated position from the orbit) in right ascension and declination were a few arc-seconds and less than one arc-second, respectively.

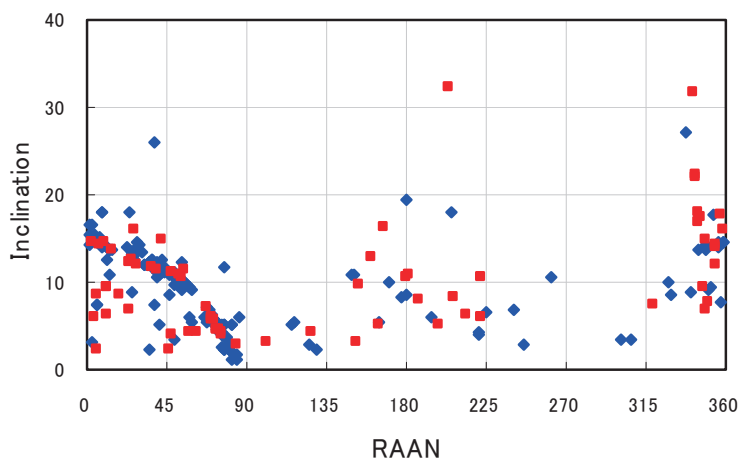

Fig. 12. Distribution of cataloged (blue column) and un-cataloged (red column) objects in the inclination-RAAN space. 


\section{Other Research Activity (Survey Observations)}

Survey observations are being carried out to improve our understanding of the space environment near GEO region. One or two inertia positions in space are observed for a single night. By observing many inertia positions for many nights, the space environment will be assumed. As described in Fig. 9, observing inertia positions requires changing the direction of the telescope many times during one night's observation. The observation position is changed every five minutes, and 18 frames are taken with a 3 -second exposure in each position. Telescope tracking is paused during the exposure so that GEO objects appear as point-like shapes on the CCD frames. About 100 areas are observed each night. All the data are analyzed with the line-identifying technique described in section 5.2. Fig.10 shows the brightness distribution of detected GEO objects from 15 nights of survey data. The blue and red columns represent cataloged and un-cataloged objects. The $x$ and $y$ axes show the brightness (magnitude) of detected objects and their number. Sample images of cataloged $\left(12^{\text {th }}\right.$ magnitude) and un-cataloged $\left(17^{\text {th }}\right.$ magnitude) objects that were detected in the survey data are shown in Fig.11. As can be seen from Fig.11, even with the line-identifying technique, fairly faint objects are detectable, although the stacking method has greater capability. The size of objects in GEO with a brightness of $16.5^{\text {th }}$ and $17.5^{\text {th }}$ magnitude is $60 \mathrm{~cm}$ and $40 \mathrm{~cm}$ in diameter, respectively. Fig. 12 is the distribution of cataloged and un-cataloged objects in inclination and RAAN space.

\section{Conclusions}

Observational techniques for the detection and orbit determination of un-cataloged GEO debris have been established. In order to detect small GEO debris, the stacking method and the line-identifying technique were developed. The major shortcoming of the stacking method will be solved by using FPGA in the near future. An efficient orbital determination method has been tested and shown to work well. Other research and development activities at the Mt. Nyukasa optical observation facility were also introduced. We would like to contribute to solving the space debris problem in the future, using these techniques.

\section{References}

1) Portree, D. S. F. and Loftus, J. P.: Orbital Debris: A Chronogy, NASA TP-1999-208856, pp.142-146, 1999.

2) Yanagisawa, T., et al: Detection of Small GEO Debris by Use of the Stacking Method, Trans. Japan Soc. Aero. Space Sci, 44 (2002), pp. 190-199.

3) Yanagisawa, T., et al: Automatic Detection Algorithm for Small Moving Objects, Publ. Astron. Soc. Japan, 57 (2005), pp. 399-408.

4) Nakajima, A., Yanagisawa, T., Kimura, T., Isobe, T., Tsujii, T., Yamamoto, M., Hoshino, T., Suzuki, M. and Futami, H.: Space Debris Observation by Ground-Based Optical Telescope, Proc. $22^{\text {nd }}$ Int. Symp. Space Technology and Science, pp. 2055-2060, 2000

5) Washio, T., Umehara, H. and Kimura, K.: An Optical Survey of Geostationary Satellites and Debris, The Review of the Communications Research Laboratory, 45, 3/4 (1999), pp. 175-180.

6) Kawase, S.: Orbit Determination Accuracy for Optically Tracked Near Synchronous Debris, Proc. $9^{\text {th }}$ Workshop Astrodynamics and Flight Mechanics, ISAS, Kanagawa, Japan, pp. 375-380, 1999

7) Umehara, H. and Kimura, K.: An optical search for near-synchronous debris: Survey to 90 degrees of right ascension, J. Jpn. Soc. Aeronaut. Space Sci, 49 (2001),pp. 1-8,

8) Umehara, H., et al: Scan by Monitoring a Pair of Points -- Optical Survey Method for Near-Geosynchronous Orbits, Trans. JSASS Space Tech. Japan, 3, (2005), pp.13-22. 